\title{
Placental prostate-specific antigen content in preeclampsia
}

\author{
Murat Can ${ }^{1 凶}$, Serefden Acıkgoz¹, Berrak Guven¹ and Ulku Ozmen Bayar ${ }^{2}$ \\ 1Department of Biochemistry; ${ }^{2}$ Department of Gynecology and Obstetrics, Faculty of Medicine, Karaelmas University, Zonguldak, Turkey
}

\begin{abstract}
Preeclampsia is a multisystem disorder that can manifest clinically with hypertension and proteinuria. Previous studies reported the presence of placental PSA in normal pregnancy but no study has been done in preeclampsia. The aim of this study was to investigate PSA content in preeclampsia. Preeclampsia was diagnosed according to the American College of Obstetricians and Gynecologists criteria. Placentas were obtained from 33 preeclamptic and 34 normotensive women. Placenta samples were homogenized and the supernatants were immediately analyzed. The tissue PSA content was measured by Immulite 2000 PSA assay. The data were analyzed with Student's $t$-test and Pearson correlation test. There was a significant difference in placental PSA content between preeclamptic and normotensive women. Placental content of PSA was higher in the preeclamptic group with intrauterine growth restriction (IUGR) than in the preeclamptic and normotensive pregnant without IUGR groups. No significant difference was found in this respect between preeclamptic and normotensive women without IUGR. In conclusion, we found that placental PSA content is elevated in preeclampsia and negatively correlated with infant birth weight. Further studies will be necessary to define the roles of PSA more precisely and to examine its effects on the pathophysiology of preeclampsia.
\end{abstract}

Key words: prostate specific antigen, preeclampsia

Received: 25 February, 2012; revised: 16 July, 2012; accepted: 30 July, 2012; available on-line: 01 August, 2012

\section{INTRODUCTION}

Preeclampsia is a multisystem disorder that can manifest clinically with hypertension and proteinuria with or without accompanying symptoms, abnormal maternal laboratory test results, intrauterine growth restriction, or reduced amniotic fluid volume (Sibai et al., 2005). Although its pathogenesis remains an enigma, it is nevertheless widely accepted that the abnormality in this disorder lies in the placenta, as the disease usually abates soon after the delivery of the placenta.

Prostate specific antigen (PSA, also known as kallikrein-related peptidase $3, \mathrm{KLK} 3$ ) is a $28-\mathrm{kDa}$ serine protease secreted as a zymogen (Lundwall et al., 2006). PSA is generally considered a biological marker of prostate cancer and raised values may also be observed in benign prostatic diseases. PSA has been shown to be expressed in many female organs such as the breast and ovary, and in amniotic fluid (Chang et al., 2011; Mardanian et al., 2011; Athanassiadou et al., 2002). Previous studies indicated that PSA is synthesized by the syncytiotrophoblast in the placenta and secreted to the amniotic fluid during pregnancy but its physiological function has not been clarified yet (Sarandakou et al., 2007).

Although the presence of placental PSA has been reported in normal pregnancy, to our knowledge no study has been done yet to determine placental PSA in preeclampsia. In this study we therefore determined the content of placental PSA in normotensive and preeclamptic pregnant women.

\section{MATERIALS AND METHODS}

Placentas were obtained from 33 preeclamptic and 34 normotensive women. The subjects were matched for age and gestation. The study was approved by the University's Ethics Committee. The placentas were collected after obtaining informed consent from all the subjects.

Preeclampsia was diagnosed when hypertension (systolic blood pressure $\geq 140 \mathrm{mmHg}$ or diastolic blood pressure $\geq 90 \mathrm{mmHg}$ ) and proteinuria ( $\geq 300 \mathrm{mg} /$ day) appeared after 20 week of gestational age in previously normotensive women, according to the American College of Obstetricians and Gynecologists criteria (ACOG criteria practice bulletin, 2002). Baseline data consisting of maternal age, gestational age, systolic blood pressure and diastolic blood pressure, Apgar scores and neonatal birth weight were recorded. Patients were exluded from the study if they had a history of diabetes, renal disease, hepatic disease, hypertension, cardiovascular illness, multiple pregnancies, hemolysis, elevated liver function, low platelets (HELLP) syndrome, autoimmune diseases, or with a fetus having structural or genetic anomaly or infections. During the period of the study, three admissions were excluded from the study. After delivery, fetal intrauterine growth restriction (IUGR) was defined as birthweight $<10$ th percentile as assessed by gestational age at delivery (Alexander et al., 1996).

Samples were taken from the central region of the placenta and immediately frozen at $-80^{\circ} \mathrm{C}$ for analysis at a later date. Tissue samples were minced and homogenized using a glass teflon homogenizer (Ultra Turrax IKA T18 Basic) as described previously by Mannello et al., 1998. The lysates were centrifuged at $9000 \times g$ at $4{ }^{\circ} \mathrm{C}$ for 30 minutes and the supernatants were immediately analyzed. PSA content was analyzed by Immulite 2000 PSA assay (Siemens Medical Solutions Diagnostics, Los Angeles, CA, USA) with an interassay CV of $4.2 \%$ and intra-assay $\mathrm{CV}$ of $3.3 \%$. The detection limit for PSA was $0.04 \mathrm{ng} /$ $\mathrm{ml}$. Urine protein levels were measured with Siemens Advia 2400 analyzer (Siemens, Deerfield, IL, USA).

e-mail: drcanmurat@yahoo.com

Abbreviations: IUGR, fetal intrauterine growth restriction; PSA, prostate specific antigen 
Table 1. Demographic and clinical data of study groups

\begin{tabular}{llll}
\hline & Preeclampsia (33) & Normotensive Pregnant (34) & $P$ value \\
\hline Age (year) & $30.2 \pm 5.5$ & $27.8 \pm 5.4$ & $>0.05$ \\
Gestational age (week) & $34.8 \pm 5.0$ & $37.5 \pm 1.5$ & $>0.05$ \\
Parity (nullipar/multipar) & $17 / 16$ & $18 / 16$ & $>0.05$ \\
Systolic blood pressure (mmHg) & $156.6 \pm 17.4$ & $110 \pm 9.9$ & $<0.05$ \\
Diastolic blood pressure (mmHg) & $95.8 \pm 9.4$ & $71.2 \pm 8.4$ & $<0.05$ \\
Infant weight (gram) & $2384 \pm 978$ & $3188 \pm 398$ & $<0.05$ \\
\hline
\end{tabular}

Statistical evaluation was done using Statistical Package for Social Science (SPSS) version 13.0 (SPSS, Chicago, USA) packet program. Datas were presented as means \pm standard deviations. The fit of quantitative variables to normal distribution was evaluated by Kolmogorov-Smirnov test. The differences between the two groups were evaluated with Student's $t$-tests for paired samples. The correlation study was performed using the Pearson correlation test.

\section{RESULTS}

In agreement with the study design, the systolic and diastolic blood pressure were higher in the preeclampsia group than in normotensive controls. Infant weight was significantly lower in preeclamptic women. No differences were found for age, gestational age or parity (nullipar/multipar ratio) between the two groups (Table 1). The mean urine total protein of preeclamptic and normotensive pregnancy was $2065 \pm 2948 \mathrm{mg} /$ day and $100 \pm 78 \mathrm{mg} /$ day.

The placental PSA content in preeclamptic and normotensive pregnancy was $315 \pm 160$ and $220 \pm 80 \mathrm{ng} / \mathrm{g}$ wet weight, respectively (Fig. 1). That difference was significant $(P<0.05)$.

The PSA content in the placental tissue of preeclamptic pregnancies with IUGR $(n=11)$, preeclamptic without IUGR $(\mathrm{n}=22)$ and normotensive $(\mathrm{n}=34)$ without IUGR was $347 \pm 57,271 \pm 137$, and $220 \pm 80 \mathrm{ng} / \mathrm{g}$ wet weight, respectively (Fig. 2). The differences between the first group and either of those without IUGR were significant $(P<0.05)$, while the difference between the two latter groups was not.

When the relationship between PSA content and clinical data was investigated, statistically significant correlations were found between PSA and systolic pressure $(P<0.01 ; \mathrm{r}=+0.379)$, diastolic pressure $(P<0.01$; $\mathrm{r}=+0.299)$, infant weight $(P<0.01 ; \mathrm{r}=-0.348)$ and gestational age $(P<0.05 ; \mathrm{r}=-0.351)$.

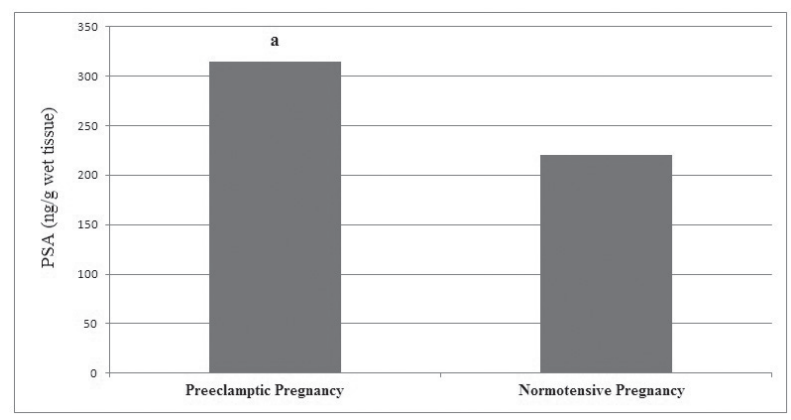

Figure 1. Placental PSA content in preeclamptic and normotensive women

(a) significant difference from normotensive pregnancy.

\section{DISCUSSION}

Human normal placenta synthesizes and secretes PSA in both free and complexed forms (Mannello et al., 1998). In vitro experiments have demonstrated that the pregnancy related steroid hormone $17 \beta$-estradiol can upregulate PSA production and secretion (Malatesta et al., 2000). PSA can cleave insulin-like growth factor-binding proteins resulting in local release of IGF-1 (Cohen et al., 1992). PSA can also specifically activate latent form of transforming growth factor beta-2 (Dallas et al., 2005). These studies showed that PSA can directly cleave or release proteins that are involved in growth stimulation and inflammation. In our study we found increased placental PSA in preeclamptic patients. The proteolytic activity of PSA on different biological substrates could in part explain the potential role of the elevated level of placental PSA in preeclampsia.

Cytotrophoblast invasion into the placental bed is necessary to convert the decidualized spiral arteries into normal uteroplacental arteries, characterized by loss of the musculoelastic structure, deposition of fibrous tissue and fibrinoid material, and re-endothelialization. Defective trophoblast invasion of myometrial spiral arteries and persistence of the arterial structure has been shown in numerous placental bed biopsy and cesarean hysterectomy specimens (Brosens et al., 2002). This contributes to a reduced placental blood flow that can result in IUGR in preeclampsia. Our study revealed on increased content of PSA in the placental tissue of preeclamptic pregnancies with IUGR compared to normotensive or preeclamptic patients without IUGR. We also found significant negative correlations between placental PSA content and infant birth weight.

To date, the association of high blood pressure with serum PSA concentration remains controversial. Han et

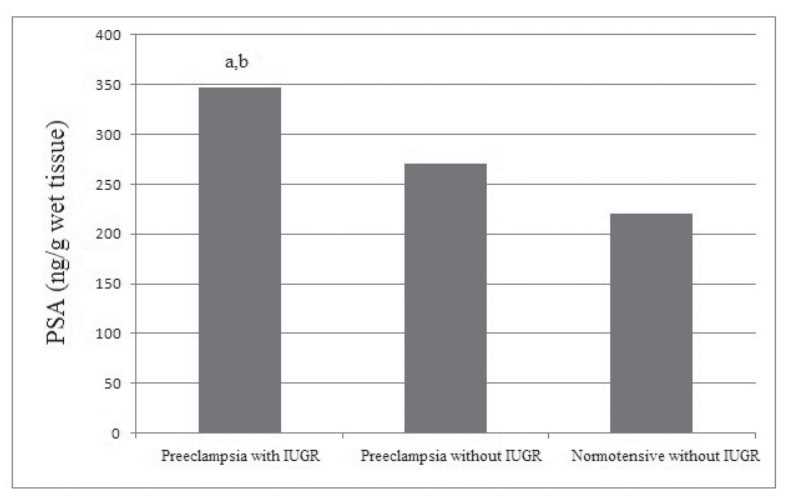

Figure 2. Placental PSA content according to IUGR (a) significant difference from preeclampsia without IUGR; (b) significant difference from normotensive without IUGR 
al. (2008) showed that elevated diastolic blood pressure was positively associated with elevated serum PSA concentrations. Jeong and coworkers (2010) obtained data from 23,601 men at 40 years or older without a history of prostate cancer and found higher serum PSA levels in men who had abnormally elevated blood pressure or who were taking antihypertensive medication. However, no significant differences in predicted geometric mean PSA concentrations were seen by blood pressure in a nationally representative cross-sectional survey of the US population (National Health and Nutrition Examination Survey 2001-2002) (Werny et al., 2006). In our study we observed a positive relationship between systolic or diastolic blood pressure and placental PSA content. Our results support previous studies which found elevated PSA levels in hypertension.

In conclusion, we found that placental PSA content is elevated in preeclampsia and negatively correlated with infant birth weight. Further studies will be necessary to define the roles of PSA more precisely and to examine their effects on the pathophysiology of preeclampsia.

\section{REFERENCES}

Alexander GR, Himes JH, Kaufman RB, Mor J, Kogan M (1996) A United States national reference for fetal growth. Obstet Gynecol. 87: 163-168.

American College of Obstetricians and Gynecologists (ACOG) Criteria practice bulletin. Diagnosis and management of preeclampsia and eclampsia. (2002) Obstet Gynecol 99: 159-167.

Athanassiadou P, Gonidi M, Liossi A, Nakopoulou L, Petrakakou E, Sakellariou V et al. (2002) Immunoreactivity of p53, PSA and bcl-2 in amniotic fluid smears from the second trimester of pregnancy. Acta Cytol 46: 440-442.

Brosens JJ, Pijnenborg R, Brosens IA (2002) The myometrial junctional zone spiral arteries in normal and abnormal pregnancies: a review of the literature. Am J Obstet Gynecol 187: 1416-1423.
Chang YF, Hung SH, Lee YJ, Chen RC, Su LC, Lai CS et al. (2011) Discrimination of breast cancer by measuring prostate-specific antigen levels in women's serum. Anal Chem 83: 5324-5328.

Cohen P, Graves HC, Peehl DM, Kamarei M, Giudice LC, Rosenfeld RG (1992) Prostate-specific antigen (PSA) is an insulin-like growth factor binding protein-3 protease found in seminal plasma. J Clin Endocrinol Metab 75: 1046-1053.

Dallas SL, Zhao S, Cramer SD, Chen Z, Peehl DM, Bonewald LF (2005) Preferential production of latent transforming growth factor beta-2 by primary prostatic epithelial cells and its activation by prostate-specific antigen. J Cell Physiol 202: 361-370.

Han JH, Choi NY, Bang SH, Kwon OJ, Jin YW, Myung SC et al. (2008) Relationship between serum prostate-specific antigen levels and components of metabolic syndrome in healthy men. Urology 72: $749-754$.

Jeong IG, Hwang SS, Kim HK, Ahn H, Kim CS (2010) The association of metabolic syndrome and its components with serum prostate-specific antigen levels in a Korean-screened population. Cancer Epidemiol Biomarkers Prev 19: 371-380.

Lundwall A, Band V, Blaber M, Clements JA, Courty Y, Diamandis EP et al. (2006) A comprehensive nomenclature for serine proteases with homology to tissue kallikreins. Biol Chem 387: 637-41.

Malatesta M, Mannello F, Luchetti F, Marcheggiani F, Condemi L, Papa S et al. (2000) Prostate-specific antigen synthesis and secretion by human placenta: a physiological kallikrein source during pregnancy. J Clin Endocrinol Metab 85: 317-321.

Mannello F, Malatesta M, Fusco E, Bianchi G, Cardinali A, Gazzanelli $G$ (1998) Biochemical characterisation and immunolocalization of prostate-specific antigen in human term placenta. Clin Chem 44: 1735-1738.

Mardanian F, Heidari N (2011) Diagnostic value of prostate-specific antigen in women with polycystic ovary syndrome. $J$ Res Med Sci 16: $999-1005$.

Sarandakou A, Protonotariou E, Rizos D (2007) Tumor markers in biological fluids associated with pregnancy. Crit Rev Clin Lab Sci 44: 151-178.

Sibai B, Dekker G, Kupfermic M (2005) Preeclampsia. Lancet 365: 785-799

Werny DM, Saraiya M, Gregg EW (2006) Prostate-specific antigen values in diabetic and nondiabetic US men, 2001-2002. Am J Epidemiol 164: 978-983. 
